\title{
Patients with Autoimmune Hepatitis Report Lower Lifetime Coffee Consumption
}

\author{
Craig Lammert $^{1}{ }^{1} \cdot$ Sai N. Chalasani ${ }^{1} \cdot$ Kelsey Green ${ }^{1} \cdot$ Elizabeth Atkinson $^{2} \cdot$ Bryan McCauley $^{2}$. \\ Konstantinos N. Lazaridis ${ }^{2}$
}

Received: 14 December 2020 / Accepted: 30 March 2021 / Published online: 30 April 2021

(c) The Author(s) 2021

\begin{abstract}
Background Coffee drinking has been associated with decreased risk of some autoimmune diseases as well liver disease and outcomes. Environmental factors, such as coffee consumption, are yet to be assessed among patients with autoimmune hepatitis (AIH).

Aim We sought to investigate the relationship between coffee consumption and risk of AIH utilizing the Genetic Repository of Autoimmune Liver Disease and Contributing Exposures (GRACE) database.

Methods Lifetime coffee drinking was collected from 358 AIH patients (cases) and 564 volunteers (controls) from primary care visits. Groups were compared utilizing the Wilcoxon rank sum test for continuous variables and the Chi-square test for discrete variables. Logistic regression was used to analyze the effects of different coffee parameters (time, frequency, and cups) after adjusting for age, sex, education, smoking status, BMI, and daily activity.

Results $24.6 \%$ of AIH patients never drank coffee compared to $15.7 \%$ of controls $(p<0.001)$, and only $65.6 \%$ were current drinkers compared with 77\% of controls $(p<0.001)$. Among "ever" coffee drinkers, AIH patients consumed fewer lifetime cups of coffee per month ( 45 vs. 47 for controls, $p<0.001)$ and spent less percentage of life drinking coffee $(62.5 \%$ vs. $69.1 \%$ for controls, $p<0.001)$. Concurrent inflammatory bowel disease was higher among AIH patients than controls $(5.7 \%$ vs. $1.2 \%, p<0.001$ ), yet did not significantly contribute to "never" coffee drinking status. The relationship between lower coffee consumption and AIH persisted even after controlling for covariates.
\end{abstract}

Conclusions Coffee consumption is lower among patients with AIH compared to controls.

Keywords Caffeine $\cdot$ Coffee $\cdot$ Environment $\cdot$ Autoimmune hepatitis $\cdot$ AIH

$\begin{array}{ll}\text { Abbreviations } \\ \text { AIH } & \text { Autoimmune hepatitis } \\ \text { GRACE } & \begin{array}{l}\text { Genetic Repository of Autoimmune Liver Dis- } \\ \text { ease and Contributing Exposures }\end{array} \\ \text { PBC } & \text { Primary biliary cholangitis } \\ \text { PSC } & \text { Primary sclerosing cholangitis } \\ \text { UC } & \text { Ulcerative colitis } \\ \text { IBD } & \text { Inflammatory bowel disease } \\ \text { CD } & \text { Crohn's disease }\end{array}$

Craig Lammert

clammert@iu.edu

1 Division of Gastroenterology and Hepatology, Indiana University School of Medicine, 702 Rotary Circle, Suite 205, Indianapolis, IN 46202, USA

2 Division of Gastroenterology and Hepatology, Mayo Clinic College of Medicine, Rochester, MN, USA

\section{Introduction}

Autoimmune hepatitis (AIH) is a chronic liver disease resulting from immune intolerance and failure of immunologic homeostasis. AIH can be diagnosed in any population demographic, yet worse outcomes have been associated with those with early disease onset, incomplete normalization of liver tests following therapy, advanced fibrosis at diagnosis, and African-Americans [1-3].

The etiology of AIH remains uncertain, but is likely established as the result of a permissive genetic architecture and several yet unknown environmental exposures. The strongest support of an environmental contribution to AIH development includes small observational studies of viruses (hepatitis A, B, C, E, Epstein-Barr, varicella zoster) and reported drug-mediated associations $[4,5]$. Drug-induced AIH has often been linked to nitrofurantoin and minocycline, yet these cases do not have the common risk alleles 
observed in idiopathic AIH [6]. Other environmental risks must be important, but assessment of other external factors remains incomplete [7].

Coffee is ubiquitous, and the benefits of this beverage have been touted since scientific literature has shown numerous health benefits. Not only has coffee been linked to reduced all-cause mortality [8], it has also has been protective in liver disease against elevated liver tests and fibrosis progression $[9,10]$. Further, coffee may be protective of some autoimmune diseases (ulcerative colitis, multiple sclerosis) [11], and recently, we showed primary sclerosing cholangitis (PSC) patients drank less coffee than controls [12].

Assessment of coffee drinking among AIH patients has not been performed. In the current study, we aimed to assess prevalence and patterns of coffee consumption among a well-described cohort of AIH patients and control group.

\section{Methods}

\section{Study Populations}

The Genetic Repository of Autoimmune Liver Disease and Contributing Exposures (GRACE) study contains consecutively recruited AIH patients from Indiana University [13]. This ongoing AIH patient repository was initiated in 2014 to collect retrospective and prospective $\mathrm{AIH}$ cases in order investigate genetic and environmental underpinnings of disease development. All AIH patients within the GRACE study had confirmed AIH per most recent clinical guidelines [14].

Controls were serially recruited from the Mayo Clinic Division of General Internal Medicine during annual visits for preventive medical examination. Control exclusion criteria included evidence of prior or current liver disease or any historical abnormal liver enzyme levels. Informed consent was obtained from all the study participants. The present study and included cohorts conform to the ethical guidelines of the 1975 Declaration of Helsinki and have been approved by the Institutional Review Board of Mayo Clinic and Indiana University.

\section{Demographic, Medical History, and Lifestyle Assessment}

Both cases and controls were provided general and coffeespecific questionnaires at time of enrollment. The general questionnaire included information regarding demographics, medical history, and lifestyle and environmental exposures (such as tobacco use). The coffee questionnaire collected details of coffee drinking. These were completed at study visit or returned via mail. The study instruments were developed by the Mayo Clinic Survey Center (MCSC). Follow-up questionnaires were resent to enrolled subjects within 2 months of recruitment in the case of non-response.

\section{Coffee Assessment}

The coffee use questionnaire included ten questions outlining current (i.e., coffee use in the past one year) and past (i.e., lifetime) habits of coffee drinking [12]. Parameters of coffee consumption were completed by totaling caffeinated and decaffeinated coffee responses. The questionnaire assessed coffee drinking using 11 frequency categories, ranging from less than one cup per month to more than 9 cups per day. It also included detailed information to define age started drinking coffee and age stopped drinking coffee, thus providing assessment of average lifetime cups of coffee per month and percentage of lifetime consuming at least one cup of coffee per month. In this study, the total years drinking coffee and cup frequency allowed the calculation of "average lifetime cups of coffee" and age and time drinking at least one cup of coffee per month allowed calculation of "percentage of lifetime drinking coffee." A "lifetime coffee status" was identified by three separate categories including current, past, and never a coffee drinker.

\section{Statistical Analysis}

Continuous variables were summarized using medians and the 25th and 75th percentiles, and $p$ values were obtained using the Wilcoxon rank sum test. $p$ values for discrete variables were obtained from the Chi-square test. Logistic regression was used to estimate the influence of the various coffee parameters either univariately or after adjusting for age at coffee survey, sex, smoking status, and higher education.

\section{Results}

\section{Demographic, Medical History, and Lifestyle}

At the time of study completion, the GRACE study was composed of 512 AIH patients and 349 (68.2\% response rate) had returned both study questionnaires. The control group included 563 participants (87\% response rate). The median time between enrollment and survey collection among AIH patients was $<1$ month. In this study, AIH patients were more likely to be younger at the time of survey (55 years vs. 66 years, $p<0.001)$ and have concurrent inflammatory bowel disease $(5.7 \%$ vs. $1.2 \%, p<0.001)$ compared to controls (Table 1). AIH patients were similar to controls in regard to percentage female sex, reporting higher education, BMI, and smoking status (smoked more than 100 cigarettes in lifetime). Daily activity level of AIH 
Table 1 Demographics and lifestyle assessment of AIH patients and controls

\begin{tabular}{lllr}
\hline & $\begin{array}{l}\text { AIH patients } \\
(n=349)\end{array}$ & $\begin{array}{l}\text { Controls } \\
(n=563)\end{array}$ & $p$ value \\
\hline Sex, \% female & $275(78.8 \%)$ & $414(73.5 \%)$ & 0.072 \\
BMI & $27.4(23.1,32.7)$ & $26.6(23.7,29.9$ & 0.126 \\
Age at coffee survey ${ }^{\mathrm{a}}$ & $55(44.0,64)$ & $66(59.0,73.0)$ & $<0.001$ \\
Diagnosis of IBD, \% diagnosis $_{\text {Higher education, \% beyond high school }}$ & $19(5.7 \%)$ & $7(1.2 \%)$ & $<0.001$ \\
$>100$ lifetime cigarettes, $n(\%)$ & $270(78.9 \%)$ & $452(81 \%)$ & 0.452 \\
\hline
\end{tabular}

${ }^{\text {a } V a r i a b l e ~ s u m m a r i z e d ~ a s ~ m e d i a n ~(f i r s t ~ q u a r t i l e, ~ t h i r d ~ q u a r t i l e) ~}$ patients was generally lower than controls $(p<0.001)$, as AIH patients had higher frequencies of no activity (13.3\%) and mild activity level $(39.8 \%)$ than controls $(6.4 \%$ and $35.2 \%$, respectively).

\section{Coffee Drinking}

AIH patients and controls were different according to lifetime coffee status (Table 2). Among AIH patients, 24.6\% reported never, $9.7 \%$ past, and $65.6 \%$ current coffee drinking, whereas $15.7 \%$ of controls reported never, $7.3 \%$ past, and $77 \%$ current coffee drinking $(p<0.001)$. A similar minority of AIH patients (36.4\%) and controls (35.9\%) had not started drinking coffee by 18 years of age. AIH patients reported they had spent $50 \%$ of their life drinking coffee (at least on average 1 cup/month) compared to $66.7 \%$ of controls $(p<0.001)$. The average lifetime cups of coffee consumed per month were lower in AIH patients compared to controls $(14$ cups $(0,45)(25$ th percentile, 75 th percentile) vs. $45(7.5,105), p<0.001)$. Considering only "ever" coffee drinkers, AIH patients reported less percent of their life drinking coffee compared to controls (62.5\% vs. $69.1 \%, p<0.001)$. "Ever" coffee drinking AIH patients also reported fewer average cups of coffee consumed per month compared to controls $(45(5,55)$ vs. $47(45,106), p<0.001)$. Observed differences in coffee use between $\mathrm{AIH}$ patients and

Table 2 Coffee consumption assessment of AIH patients and controls (A) and coffee consumption assessment of AIH patients and controls among "ever" drinkers (B)

\begin{tabular}{llc}
$\begin{array}{l}\text { AIH patients } \\
(n=349)\end{array}$ & $\begin{array}{l}\text { Controls } \\
(n=563)\end{array}$ & $p$ value \\
\hline
\end{tabular}

(A)

$\%$ of life drinking coffee ${ }^{\mathrm{a}}$

Coffee drinker at age 18, $n(\%)$

Lifetime cups of coffee/month (caffeinated and decaffeinated $)^{\mathrm{a}}$

Lifetime coffee status

Never coffee drinker, $n(\%)$

Past coffee drinker, $n(\%)$

Current coffee drinker, $n(\%)$

\section{$50(2.2,69.1)$ \\ $127(36.4 \%)$ \\ $14(0,46)$}

$86(24.6 \%)$

$34(9.7 \%)$

$229(65.6 \%)$

AIH patients

$(n=263)$
$66.7(34.3,73.8)$
$202(35.9 \%)$
$47(8,105)$

$88(15.7 \%)$

$41(7.3 \%)$

$433(77 \%)$

Controls

$(n=474)$

$\begin{array}{lc}69.1(57.1,75.3) & <0.001 \\ 202(42.6 \%) & 0.14 \\ 47(45,106) & <0.001 \\ & 0.066 \\ 0 & \\ 41(8.6 \%) & \\ 433(91.4) & \end{array}$

$<0.001$

0.89

$<0.001$

$<0.001$

$p$ value
(B)
$\%$ of life drinking coffee ${ }^{\mathrm{a}}$

Coffee drinker at age $18, n(\%)$

Lifetime cups of coffee/month (caffeinated and decaffeinated $)^{\mathrm{a}}$

Never coffee drinker, $n(\%)$

Past coffee drinker, $n(\%)$

Current coffee drinker, $n(\%)$
Lifetime coffee status

$62.5(39,71.2)$
$127(48.3 \%)$
$45(5,55)$

0

$34(12.9 \%)$ $229(87.1 \%)$
${ }^{a}$ Variable summarized as median (first quartile, third quartile) 
controls remained different in models including age, sex, higher education, significant smoking exposure, and BMI (data not shown).

\section{AlH, Inflammatory Bowel Disease, and Lifetime Coffee Status}

We completed additional analysis to identify any contribution of IBD on the observation of decreased coffee drinking in AIH patients. At the time of study enrollment, only $5.7 \%$ of AIH patients reported a diagnosis of inflammatory bowel disease (IBD) (controls 1.2\%, $p<0.001$ ). The majority of concurrent IBD cases were ulcerative colitis (89.4\%). Among AIH patients with ulcerative colitis (AIH-UC), only two reported proctocolectomies that were completed prior to study enrollment.

AIH diagnosis date was not available in $38 \%$ of AIH patients. Patients that never drank coffee were diagnosed at a younger age compared to those that ever drank coffee $(44$ years $(32,50)$ vs. $53(41,60), p<0.001)$ (Table 3$)$. There was no difference in age of AIH diagnosis among AIH patients with IBD (AIH-UC) according to coffee status. Among "ever" coffee drinkers, 95\% were AIH patients without IBD, 4.6\% with AIH-UC, and 0.4\% AIH-CD. "Never" coffee drinkers included 93\% AIH patients without IBD, $5.8 \%$ AIH-UC, and $1.2 \%$ AIH-CD.

\section{Discussion}

This study provides the first assessment of coffee drinking in a large cohort of well-phenotyped AIH patients within the GRACE study at Indiana University [13]. The adjusted odds of AIH were lower for current coffee drinkers $(\mathrm{OR}=0.66)$ and those consuming more average cups of coffee per month $(\mathrm{OR}=0.88$ per 20 cup increase). Furthermore, we observed a lower median age of diagnosis of AIH among both AIH patients with and without IBD that ever drank coffee relative to never coffee drinking AIH patients.

We have utilized the same coffee consumption instrument and observed a similar protective relationship of coffee drinking among PSC patients compared to controls, but not in primary biliary cholangitis (PBC) patients [12]. Autoimmune liver diseases (AIH, PSC, and PBC) are clinically heterogeneous, but foundationally are the result of interplay between complex genetic and environmental factors. Known genetic risks among AIH patients are limited because there has only been one genome-wide association study completed to date [15]. Similar to genetic risks in PSC and PBC, AIH has strong association with HLA locus variants and has shown enrichment among multiple immunoregulatory genes. Less than $20 \%$ of disease heritability has been accounted for in PSC and PBC with GWAS studies [16], and approximately $50 \%$ of disease susceptibility may be related to environmental contributions [17]. Beyond viral and compound-specific AIH triggers, no widespread environmental factors have been consistently linked to AIH [7]. This contrasts with PSC and PBC, where disease risk has been repeatedly linked to factors such as tobacco smoking and urinary tract infections $[18,19]$. In our study, there was no significant association between AIH and smoking (Table 1). The importance of environment in among PSC and PBC has been further substantiated by historical data of molecular mimicry between a list of bacterial pathogenetic factors, xenobiotic modification of epitopes, as well as clustering regionally and around superfund sites [20].

Coffee contains a diverse group of compounds capable of exerting physiologic effects that can shape responses from innate and adaptive immune systems, modify LPS-triggered inflammatory responses and cytokine signaling, work to scavenge free radicals, induce the activation DNA repair and detoxification enzymes, and function as anti-mutagens $[11,21-23]$. All or few of these cellular responses may be central to the influence of coffee among autoimmune diseases, where both risk and protection have been observed among coffee drinkers. Coffee has been linked to increased risk of rheumatoid arthritis and type I diabetes mellitus, yet reported to provided protection against multiple sclerosis, ulcerative colitis and Parkinson's disease [11, 24]. Women with high and low volume of coffee drinking were recently shown to have differential gene expression data [25]. Nearly 300 genes were differentially expressed between groups, and many were directed at the influence of both inflammatory and metabolic pathways. The bioactive molecule(s) within

Table 3 AIH and concurrent diagnosis of IBD according to lifetime coffee status: never and ever coffee drinking

\begin{tabular}{|c|c|c|c|c|c|c|}
\hline & \multicolumn{2}{|c|}{$\begin{array}{l}\text { Never a coffee drinker } \\
n=86\end{array}$} & \multicolumn{2}{|c|}{$\begin{array}{l}\text { Ever a coffee drinker } \\
N=263\end{array}$} & \multirow[t]{2}{*}{$p$ value $(n)$} & \multirow[t]{2}{*}{$p$ value (age) } \\
\hline & $n$ & Age* & $n$ & Age* & & \\
\hline AIH without IBD & $80(93 \%)$ & $44(33.8,50.2)$ & $250(95 \%)$ & $53(42,60)$ & 0.47 & $<0.001$ \\
\hline AIH-UC & $5(5.8 \%)$ & $21(19.5,30.5)$ & $12(4.6 \%)$ & $44(35,53.5)$ & 0.64 & 0.11 \\
\hline AIH-CD & $1(1.2 \%)$ & $\mathrm{n} / \mathrm{a}$ & $1(0.4 \%)$ & $32(32,32)$ & 0.4 & $\mathrm{n} / \mathrm{a}$ \\
\hline Colectomy among AIH-UC & $1(1.2 \%)$ & $\mathrm{n} / \mathrm{a}$ & $1(0.4 \%)$ & $\mathrm{n} / \mathrm{a}$ & 0.4 & $\mathrm{n} / \mathrm{a}$ \\
\hline
\end{tabular}

*Age at AIH diagnosis summarized as median in years (first quartile, third quartile) 
coffee that provides net immunomodulatory benefit in autoimmune disease remains unknown. However, any of the bioactive compounds found in coffee such as caffeine, cafestol, kahweol, or polyphenols may contribute to this effect [26, 27].

Our data suggest a dose-dependent relationship between coffee and risk of AIH development. Odds of AIH were reduced for every increase in $\%$ of life drinking coffee per 20 cups $(\mathrm{OR}=0.9)$ as well as for every increase in 20 cups of coffee/month $(\mathrm{OR}=0.87)$. Dose-dependent coffee benefit has been observed in reduction of all-cause mortality [28] as well as mortality associated with other chronic diseases [29]. Disease modification has also been observed with coffee including reduced risk of cirrhosis development among chronic liver disease patients [30] and even delay in onset of Parkinson's disease [31]. In our study, we observed AIH patients that never drank coffee had an earlier median age of disease onset compared to AIH patients that ever drank coffee (Table 3) albeit a significant missing proportion with available age of diagnosis. Animal and cellular models have identified a number of plausible bioactive compounds responsible, yet in the real world even the type of coffee [32], brewing technique [33], and filter use may have dramatic impact on coffee makeup [34].

The beneficial attributes of coffee have been reportedly prevalent among chronic liver diseases. Nearly 30 years ago, a sentinel study of heavy drinkers first revealed the risk of liver cirrhosis among concurrent coffee drinkers was one-fifth the risk of non-coffee drinkers [9]. Subsequently, coffee was associated with more favorable liver tests among high-risk patients [10] and decreased mortality in patients with cirrhosis [35]. We were unable to assess the relationship between disease progression and coffee use in this study given the cross-sectional approach. However, $30 \mathrm{AIH}$ patients in this study had undergone transplant by time enrollment. Transplanted AIH patients were no different from AIH patients without transplant according to percent life drinking coffee, drinking status at age 18, average cups per month, and lifetime coffee drinking status (data not shown). Limitations within this study include self-reporting of coffee habits and memory bias inherent in considering a lifetime of coffee use, as well as a low population of patients and controls that have previously drank coffee then stopped. Among our control group that has been used previously to examine other chronic liver diseases, $77 \%$ of individuals were current coffee drinkers. This is quite similar to the $75 \%$ of current drinkers over the age of 20 years observed in the 2003 to 2012 National Health and Nutrition Examination Survey data [36].

Further, coffee use could encompass a wide range of products, and we were unable to assess for this variability, how beverages were prepared, or the fluid ounces per cup. We believe the observed findings are valid as the survey tool has performed well among PSC and PBC patients, is biologically plausible, and includes an excellent response rate in the well-phenotyped AIH study cohort. Furthermore, we were able to adjust for important confounders such as smoking, age, education level, and BMI.

In conclusion, we observed less coffee consumption among AIH patients compared to a control population. Future studies examining a relationship between coffee consumption and liver-related outcomes and transplant-free survival in AIH should be considered.

Author's contribution CL was involved in assembly/interpretation of data and drafted and revised the manuscript. KG collected the coffee instrument, checked the data, and reviewed the manuscript. BM and EJA were involved in statistical analysis and critical revision of statistics and results. SC was involved in assembly/interpretation of data and drafted and reviewed the manuscript. KNL was involved in conception of study and design, collection, and assembly of data and drafted and approved the final manuscript version.

Funding CL was supported by NIDDK K23DK11456.

\section{Declarations}

Conflict of interest All included authors declare no outside interests that are directly or significantly related to this paper.

Open Access This article is licensed under a Creative Commons Attribution-NonCommercial 4.0 International License, which permits any non-commercial use, sharing, adaptation, distribution and reproduction in any medium or format, as long as you give appropriate credit to the original author(s) and the source, provide a link to the Creative Commons licence, and indicate if changes were made. The images or other third party material in this article are included in the article's Creative Commons licence, unless indicated otherwise in a credit line to the material. If material is not included in the article's Creative Commons licence and your intended use is not permitted by statutory regulation or exceeds the permitted use, you will need to obtain permission directly from the copyright holder. To view a copy of this licence, visit http://creativecommons.org/licenses/by-nc/4.0/.

\section{References}

1. Verma S, Torbenson M, Thuluvath PJ. The impact of ethnicity on the natural history of autoimmune hepatitis. Hepatology. 2007;46:1828-1835.

2. Ngu JH, Gearry RB, Frampton CM, Stedman CA. Predictors of poor outcome in patients with autoimmune hepatitis: a populationbased study. Hepatology. 2013;57:2399-2406.

3. Feld JJ, Dinh H, Arenovich T, Marcus VA, Wanless IR, Heathcote EJ. Autoimmune hepatitis: effect of symptoms and cirrhosis on natural history and outcome. Hepatology. 2005;42:53-62.

4. Illing PT, Vivian JP, Dudek NL, et al. Immune self-reactivity triggered by drug-modified HLA-peptide repertoire. Nature. 2012;486:554-558. 
5. Tanaka H, Tujioka H, Ueda H, Hamagami H, Kida Y, Ichinose M. Autoimmune hepatitis triggered by acute hepatitis A. World J Gastroenterol. 2005;11:6069-6071.

6. de Boer YS, Kosinski AS, Urban TJ, et al. Features of autoimmune hepatitis in patients with drug-induced liver injury. Clin Gastroenterol Hepatol. 2017;15:103-112.e102.

7. Ngu JH, Gearry RB, Frampton CM, Stedman CA. Autoimmune hepatitis: the role of environmental risk factors: a populationbased study. Hepatol Int. 2013;7:869-875.

8. Freedman ND, Park Y, Abnet CC, Hollenbeck AR, Sinha R. Association of coffee drinking with total and cause-specific mortality. N Engl J Med. 2012;366:1891-1904.

9. Klatsky AL, Armstrong MA. Alcohol, smoking, coffee, and cirrhosis. Am J Epidemiol. 1992;136:1248-1257.

10. Ruhl CE, Everhart JE. Coffee and caffeine consumption reduce the risk of elevated serum alanine aminotransferase activity in the United States. Gastroenterology. 2005;128:24-32.

11. Sharif K, Watad A, Bragazzi NL, Adawi M, Amital H, Shoenfeld Y. Coffee and autoimmunity: more than a mere hot beverage! Autoimmun Rev. 2017;16:712-721.

12. Lammert C, Juran BD, Schlicht E, et al. Reduced coffee consumption among individuals with primary sclerosing cholangitis but not primary biliary cirrhosis. Clin Gastroenterol Hepatol. 2014;12:1562-1568.

13. Lammert C, Comerford M, Love J, Bailey JR. Investigation gone viral: application of the social mediasphere in research. Gastroenterology. 2015;149:839-843.

14. EASL Clinical Practice Guidelines. Autoimmune hepatitis. $J$ Hepatol. 2015;63:971-1004.

15. de Boer YS, van Gerven NM, Zwiers A, et al. Genome-wide association study identifies variants associated with autoimmune hepatitis type 1. Gastroenterology. 2014;147:443-452.e445.

16. Reichert MC, Hall RA, Krawczyk M, Lammert F. Genetic determinants of cholangiopathies: molecular and systems genetics. Biochim Biophys Acta Mol Basis Dis. 2018;1864:1484-1490.

17. Lammert C. Genetic and environmental risk factors for autoimmune hepatitis. Clin Liver Dis (Hoboken). 2019;14:29-32.

18. Juran BD, Lazaridis KN. Environmental factors in primary biliary cirrhosis. Semin Liver Dis. 2014;34:265-272.

19. Eaton JE, Juran BD, Atkinson EJ, et al. A comprehensive assessment of environmental exposures among 1000 North American patients with primary sclerosing cholangitis, with and without inflammatory bowel disease. Aliment Pharmacol Ther. 2015;41:980-990.

20. Ala A, Stanca CM, Bu-Ghanim M, et al. Increased prevalence of primary biliary cirrhosis near Superfund toxic waste sites. Hepatology. 2006;43:525-531.

21. Weber L, Hammoud Mahdi D, Jankuhn S, Lipowicz B, Vissiennon C. Bioactive plant compounds in coffee charcoal (Coffeae carbo) extract inhibit cytokine release from activated human THP-1 macrophages. Molecules. 2019;24:4263.

22. Hang D, Kvaerner AS, Ma W, et al. Coffee consumption and plasma biomarkers of metabolic and inflammatory pathways in US health professionals. Am J Clin Nutr. 2019;109:635-647.
23. Nehlig A, Debry G. Potential genotoxic, mutagenic and antimutagenic effects of coffee: a review. Mutat Res. 1994;317:145-162.

24. Paul KC, Chuang YH, Shih IF, et al. The association between lifestyle factors and Parkinson's disease progression and mortality. Mov Disord. 2019;34:58-66.

25. Barnung RB, Nøst HT, Ulven SM, Skeie G, Olsen SK. Coffee consumption and whole-blood gene expression in the Norwegian Women and Cancer Post-Genome Cohort. Nutrients. 2018;10:1047.

26. Bonita JS, Mandarano M, Shuta D, Vinson J. Coffee and cardiovascular disease: in vitro, cellular, animal, and human studies. Pharmacol Res. 2007;55:187-198.

27. Modi AA, Feld JJ, Park Y, et al. Increased caffeine consumption is associated with reduced hepatic fibrosis. Hepatology. 2010;51:201-209.

28. Li Q, Liu Y, Sun X, et al. Caffeinated and decaffeinated coffee consumption and risk of all-cause mortality: a dose-response metaanalysis of cohort studies. J Hum Nutr Diet. 2019;32:279-287.

29. Kim Y, Je Y, Giovannucci E. Coffee consumption and all-cause and cause-specific mortality: a meta-analysis by potential modifiers. Eur J Epidemiol. 2019;34:731-752.

30. Kennedy OJ, Roderick P, Buchanan R, Fallowfield JA, Hayes PC, Parkes J. Systematic review with meta-analysis: coffee consumption and the risk of cirrhosis. Aliment Pharmacol Ther. 2016;43:562-574.

31. Yahalom G, Rigbi A, Israeli-Korn S, et al. Age at onset of Parkinson's disease among Ashkenazi jewish patients: contribution of environmental factors, LRRK2 p.G2019S and GBA p.N370S mutations. J Parkinsons Dis. 2020;10:1123-1132.

32. Rothwell JA, Loftfield E, Wedekind R, et al. A metabolomic study of the variability of the chemical composition of commonly consumed coffee brews. Metabolites. 2019;9:17.

33. Tverdal A, Selmer R, Cohen JM, Thelle DS. Coffee consumption and mortality from cardiovascular diseases and total mortality: does the brewing method matter? Eur J Prev Cardiol. 2020;27:1986-1993.

34. Rendon MY, Dos Santos Scholz MB, Bragagnolo N. Physical characteristics of the paper filter and low cafestol content filter coffee brews. Food Res Int. 2018;108:280-285.

35. Tverdal A, Skurtveit S. Coffee intake and mortality from liver cirrhosis. Ann Epidemiol. 2003;13:419-423.

36. Loftfield E, Freedman ND, Dodd KW, et al. Coffee drinking is widespread in the United States, but usual intake varies by key demographic and lifestyle factors. J Nutr. 2016;16:1762-1768.

Publisher's Note Springer Nature remains neutral with regard to jurisdictional claims in published maps and institutional affiliations. 